\title{
Radiological Growth Patterns of Prolactinomas and Nonfunctioning Adenomas
}

\author{
Syed Ali Imran, * Jai Shankar, * Andrea L.O. Hebb, Sidney E. Croul, \\ David B. Clarke
}

\begin{abstract}
Objectives: To compare growth patterns of nonfunctioning and prolactin-producing pituitary macroadenomas, and to find whether their specific growth patterns are associated with clinically significant effects on vision. Materials and Methods: From our comprehensive provincial neuropituitary registry, we retrospectively identified 35 randomly selected patients each with nonfunctioning adenomas and prolactinomas $>10 \mathrm{~mm}$ in any dimension. MRI scans were analyzed to determine the superior and inferior growth, volume, and maximum craniocaudal height of the adenomas. Patients underwent visual field testing at diagnosis. Continuous variables were compared using Student's $t$ test, the Mann-Whitney $U$ test, and ANOVA. Categorical variables were compared using the chi-square test. Results: The mean height of prolactinomas $(23.2 \pm 11.3 \mathrm{~mm})$ was similar to nonfunctioning adenomas $(22.3 \pm 9.3 \mathrm{~mm}, p=0.8)$, and so were mean tumor volumes (prolactinoma $=5.9 \pm 8 \mathrm{ml}$ vs. nonfunctioning adenoma $=4.8 \pm 5 \mathrm{ml}, p=0.47$ ). However, the mean suprasellar growth for prolactinomas was $2.9 \pm 5.3 \mathrm{~mm}$ and $7.3 \pm 4.7 \mathrm{~mm}$ for nonfunctioning adenomas $(p<0.001)$, and the mean infrasellar growth was $10.2 \pm 8.0$ and $5.0 \pm 6.6 \mathrm{~mm}$, respectively $(p=0.04)$. The inferior growth pattern of prolactinomas was associated with a significantly lower likelihood of having visual field abnormalities (11.4 vs. 57.1\%, $p<0.001)$. Conclusions: Prolactinomas have predominantly inferior growth compared to nonfunctioning adenomas and are less likely to cause vision changes.
\end{abstract}

RÉSUMÉ: Profils radiologiques de croissance des prolactinomes et des adénomes non fonctionnels. Objectifs: Le but de l'étude était de comparer le profil de croissance de macroadénomes pituitaires non fonctionnels et de prolactinomes et de déterminer si leurs profils de croissance sont associés à des effets significatifs au point de vue clinique sur la vision. Méthodologie : Nous avons identifié rétrospectivement 35 patients atteints d'adénomes non fonctionnels et de prolactinomes de plus de $10 \mathrm{~mm}$ dans au moins une dimension, choisis au hasard dans notre registre provincial complet de pathologies neuropituitaires. Nous avons analysé les scans d'IRM afin de déterminer la croissance supérieure et inférieure, le volume et la hauteur crâniocaudale maximale des adénomes. Les patients avaient subi un champ visuel au moment du diagnostic. Nous avons comparé les variables continues au moyen du test t de Student, du test U de Mann-Whitney et de l'ANOVA. Nous avons comparé les variables qualitatives au moyen du chi-carré. Résultats : La hauteur moyenne et le volume moyen des prolactinomes (hauteur $23,2 \pm 11,3 \mathrm{~mm}$; volume $5,9 \pm 8 \mathrm{ml}$ ) étaient similaires à ceux des adénomes non fonctionnels (hauteur 22,3 $\pm 9,3 \mathrm{~mm}, \mathrm{p}=0,8$; volume $4,8 \pm 5 \mathrm{ml}, \mathrm{p}=0,47$ ). Cependant, la croissance sus-sellaire moyenne des prolactinomes était de 2,9 $\pm 5,3 \mathrm{~mm}$ et celle des adénomes non fonctionnels de $7,3 \pm 4,7 \mathrm{~mm}(\mathrm{p}<0,001)$ et leur croissance sous-sellaire était de 10,2 $\pm 8,0$ et 5,0 $\pm 6,6 \mathrm{~mm}$ respectivement $(\mathrm{p}=0,04)$. Le profil de croissance inférieur des prolactinomes était associé à une probabilité moindre d'anomalies du champ visuel $(11,4 \%$ par rapport à $57,1 \%, \mathrm{p}<0,001)$. Conclusions : Les prolactinomes ont une croissance généralement moindre que les adénomes non fonctionnels et sont moins susceptibles de causer des altérations de la vision.

Keywords: Pituitary tumors, MRI, tumor volume, visual field

doi:10.1017/cjn.2017.203

Can J Neurol Sci. 2017; 44: 508-513

\section{INTRODUCTION}

Nonfunctioning adenomas (NFAs) and prolactinomas (PRLomas) are the two most common varieties of pituitary adenoma (PA). A recent population-based survey from North America ${ }^{1}$ reported that NFAs and PRLomas constituted 48 and $41 \%$ of all PAs, respectively. These data are in agreement with other studies which showed that NFAs and PRLomas are the commonest PAs. ${ }^{2,3}$ A significant proportion of patients with either an NFA (70\%) or PRLoma (39\%) present with macroadenomas, defined as tumors $>10 \mathrm{~mm}$ in size. ${ }^{1}$ However, these macroadenomas behave differently in the sense that NFAs generally present later in life with
From the Division of Endocrinology and Metabolism, Dalhousie University, Halifax, Nova Scotia, Canada (SAI, DBC); Department of Diagnostic Radiology, Division of Neuroradiology, Dalhousie University, QEII Health Sciences Centre, Halifax, Nova Scotia, Canada (JS); Division of Neurosurgery, Dalhousie University, QEII Health Sciences Centre, Halifax, Nova Scotia, Canada (ALOH, DBC); Department of Pathology and Laboratory Medicine, Dalhousie University, QEII Health Sciences Centre, Halifax, Nova Scotia, Canada (SEC).

* S.A. Imran and J. Shankar are joint first authors.

Received November 7, 2016. Final Revisions Submitted April 4, 2017. DAte of ACCEPTANCE APRIL 13, 2017.

Correspondence to: David B. Clarke, Division of Neurosurgery, Dalhousie University, Nova Scotia Health Authority, QEII Health Sciences Centre, 1796 Summer Street, Suite 3806, Halifax, NS, B3H 3A7, Canada. E-mail: d.clarke@dal.ca 
headache, with visual field (VF) defects, and as incidental lesions. In contrast, PRLomas occur in younger individuals and present with reproductive abnormalities in most women, whereas in men they typically present with reproductive abnormalities, with headache, and as incidental findings. ${ }^{1}$ To date, no study has examined whether these distinct clinical presentations can be explained by differences in the growth propensities of these PAs. To address this question, we compared the radiological growth patterns of NFAs and PRLomas and their impact on VF abnormalities.

\section{Materials ANd Methods}

Written consents were obtained from all patients prior to enrollment. The study was approved by our institutional research board. All patients were followed by a single team comprising an endocrinologist and neurosurgeon as well as specialized nurses from endocrinology and neurosurgery. From our prospectively kept database of 1,005 pituitary macroadenomas from November of 2005 to December of 2013, we randomly selected 35 patients each with PRLomas and NFAs that presented as macroadenomas. The number of patients selected was based on the power calculation as discussed in the Statistical Analysis section below. Macroadenomas were defined as a PA $>10 \mathrm{~mm}$ in any dimension as measured on the presenting MRI. PRLomas were defined as macradenomas associated with persistently elevated prolactin (which was in keeping with the size of the tumor), the presence of symptoms related to high prolactin, and an absence of such secondary causes of hyperprolactinemia as hypothyroidism, chronic kidney disease, hepatic cirrhosis, and medications known to raise prolactin. PRLomas associated with clinical or biochemical evidence of excessive growth hormone production (mixed somatolactotrophic tumors) were excluded. NFAs were defined as macroadenomas with no clinical and/or biochemical evidence of hormonal oversecretion and serum prolactin $<100 \mu \mathrm{g} / \mathrm{L}$ (normal range $=2.1-17.7 \mu \mathrm{g} / \mathrm{L}$ in males and $2.8-29.2 \mu \mathrm{g} / \mathrm{L}$ in females). For all patients who underwent surgery, tissue diagnosis was the primary method for making the diagnosis. Patients had VF testing (Humphrey or Goldmann), ${ }^{4,5}$ including at the time of first evaluation, as part of our clinical protocol.

\section{Imaging Analysis}

All MRI scans were analyzed by a specialist neuroradiologist who was blinded to the clinical diagnosis. Patients with a PA underwent MRI using our sella imaging protocol, which includes $\mathrm{T} 2$ coronal as well as pre- and postcontrast T1 sagittal and coronal images through the sella with a slice thickness of $2.5 \mathrm{~mm}$. For the present study, we selected the postcontrast T1 sagittal images (T1 sagittal image when postcontrast image was not available) through the sella that showed the maximum craniocaudal extent of the tumor (Figures 1 and 2). The T2 coronal images were used to confirm the maximum craniocaudal extent of the tumor. On the sagittal image, a line tangential to the posterior $1 \mathrm{~cm}$ of the planum sphenoidale served as a baseline (Figures 1, 2b, and 2d). The posterior $1 \mathrm{~cm}$ of the planum sphenoidale was selected for our baseline due to known variability in the shape and slope of the planum sphenoidale. ${ }^{6}$ The superior and inferior extent of the tumor were measured in reference to this baseline (Figures 1, 2b, and $2 \mathrm{~d}$ ). Tumor above this baseline was defined as suprasellar growth (Figures 1, 2b, and 2d), and tumor below this baseline minus $10 \mathrm{~mm}$ was defined as infrasellar growth (Figures 1, 2b, and

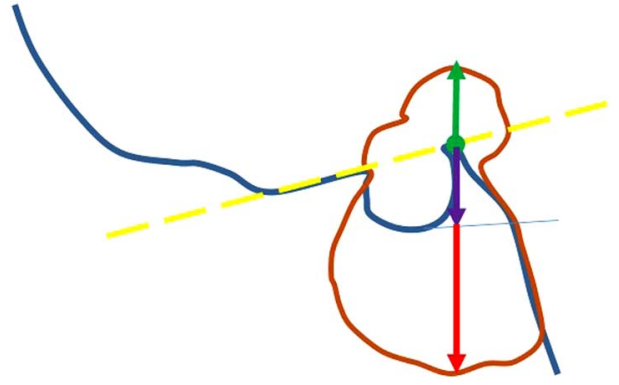

Figure 1: Demonstration of the measurements used to assess pituitary macroadenomas. A line diagram outlines the anterior base of skull, sella, and clivus (blue), tumor outline (brown), superior aspect of the sella drawn tangential to the posterior $1 \mathrm{~cm}$ of planum sphenoidale and considered the reference baseline for measurements (yellow), suprasellar growth (green), depth of sella (purple), and infrasellar growth (red).

2d). The vertical diameter of the sella ranges from 4 to $12 \mathrm{~mm},{ }^{7-10}$ and, for simplicity of calculation, we considered this to be $10 \mathrm{~mm}$ in our study. This $10 \mathrm{~mm}$ was subtracted to account for the average depth of the sella, as the floor of the sella is indistinct in many of these tumors (Figures 1 and $2 b$ ).

The difference between supra- and infrasellar growth was calculated and analyzed. Tumor volumes were calculated using the formula of an ellipsoid $(V=4 / 3 \times \pi \times[a \times b \times c])$; where $a$, $b$, and $c$ are the three radii of depth (sagittal plane), width (coronal plane), and height (coronal plane) in $\mathrm{mm}^{11}$

\section{Statistical Analysis}

To calculate the sample size, we initially conducted a pilot study of 10 randomly selected patients each with a PRLoma or NFA (all tumors $\geq 10 \mathrm{~mm}$ ). For a study power of $80 \%$ and an $\alpha$ of 0.05 , the final sample size was calculated to detect significant differences in suprasellar growth, infrasellar growth, and the difference between supra- and infrasellar growth; the estimated sample size calculations were 36,70 , and 22 , respectively. To have adequate power to find significant differences among the three means, we randomly selected 70 patients (35 NFA and 35 PRLoma) for our study using a computer-generated random number table.

The descriptive statistical analysis was performed to compare the demographic characteristics of patients. Continuous variables were compared using Student's t test, the Mann-Whitney $U$ test, and analysis of variance (ANOVA). Categorical variables were compared using the chi-square test. A $p$ value $<0.05$ was considered significant, and sensitivity, specificity, positive predictive values (PPVs), and negative predictive values (NPVs) were calculated for the suprasellar growth pattern, the infrasellar growth pattern, and the difference between supra- and infrasellar growth. Analyses were conducted using Excel 2013, GraphPad Prism (v. 6.00 for Windows, GraphPad Software, La Jolla, California, USA; www.graphpad.com), and IBM SPSS (v. 22.0 for Windows, IBM Corporation, Armonk, New York).

\section{RESULTS}

\section{Patients}

The demographic, clinical, and radiological data are summarized in Table 1 . There were more male patients-26 (74\%) and 
(a)

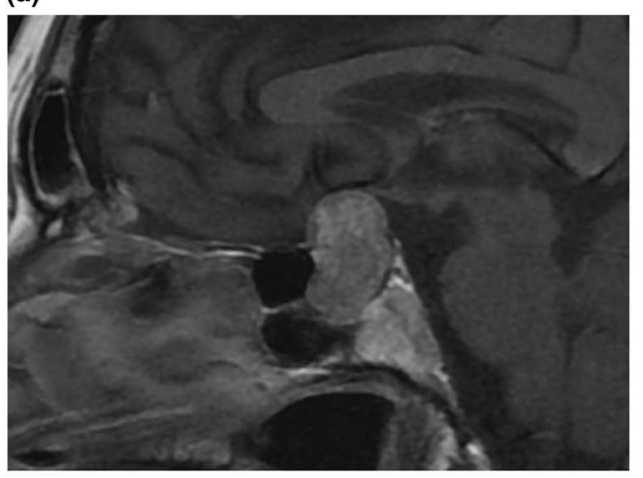

(c)

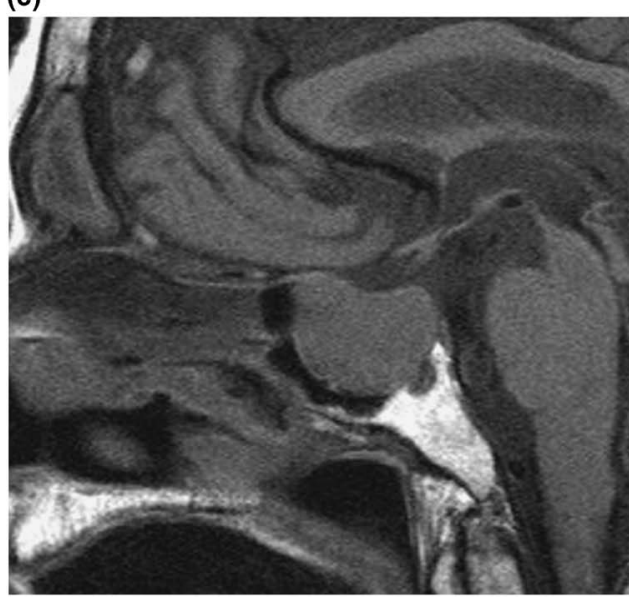

(b)

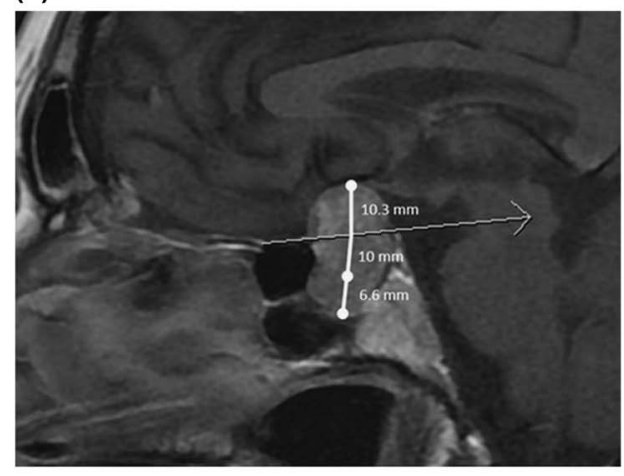

(d)

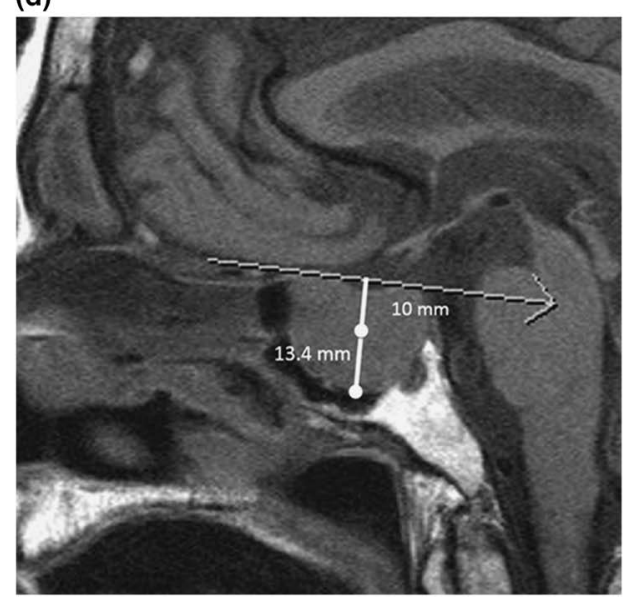

Figure 2: (a) Post-gadolinium-enhanced T1 sagittal image (in this case, an NFA with preferential suprasellar growth). (b) Reference baseline marked as an arrow; suprasellar measurement (10.3 mm); depth of sella (set at $10 \mathrm{~mm}$ ); and infrasellar measurement $(6.6 \mathrm{~mm})$. (c) Post-gadolinium-enhanced T1 sagittal image (in this case, a PRLoma with preferential infrasellar growth). (d) Reference baseline marked as an arrow; depth of sella (set at $10 \mathrm{~mm})$ and infrasellar measurement $(13.4 \mathrm{~mm})$.

$22(63 \%)$ in the PRLoma and NFA groups, respectively-with no between-groups differences $(p=0.3)$. The mean age at presentation for the PRLoma group was $49.4 \pm 17.4$ years, which was significantly lower than that for the NFA group: $65.1 \pm 13.3$ years $(p<0.01)$. Mean serum prolactin level in the PRLoma group was $1,442 \mu \mathrm{g} / \mathrm{L}$ (range $=136-7,000)$. A total of two patients in the PRLoma group underwent transsphenoidal surgery (TSS),

Table 1: Demographic, clinical, and radiological information on patients with NFAs and PRLomas

\begin{tabular}{l|c|c|c}
\hline Parameters & NFA & PRLoma & $p$ value \\
\hline Total patients & 35 & 35 & \\
\hline Male & $22(63 \%)$ & $26(74 \%)$ & 0.3 \\
\hline Mean age, years & $65.1 \pm 13.3$ & $49.4 \pm 17.4$ & $<0.001$ \\
\hline Sagittal craniocaudal diameter, mm & $22.3 \pm 9.3$ & $23.2 \pm 11.3$ & 0.73 \\
\hline Tumor volume, ml & $4.8 \pm 5$ & $5.94 \pm 8$ & 0.47 \\
\hline Visual field defect at diagnosis & $20(57 \%)$ & $4(11 \%)$ & $<0.001$ \\
\hline Suprasellar growth, mm & $7.3 \pm 4.7$ & $2.9 \pm 5.3$ & $<0.001$ \\
\hline Infrasellar growth, mm & $5.0 \pm 6.6$ & $10.2 \pm 8.0$ & 0.004 \\
\hline Supra- minus infrasellar growth & 2.3 & -7.2 & $<0.001$ \\
\hline
\end{tabular}

whereas 20 patients in the NFA group underwent TSS. All other patients were either medically treated (PRLoma) or remained under active surveillance. The primary presenting features for PRLoma were headache and reproductive symptoms; for NFA they were headache and incidental finding. The indication for surgery in the PRLoma group was an inability to tolerate medical therapy, whereas NFA patients underwent surgery because of VF abnormalities at presentation. One NFA patient had abnormal VF testing at presentation, but surgery was withheld due to multiple comorbidities and high surgical risk.

\section{Radiological Growth Pattern}

The data regarding radiological measurements are summarized in Table 1 . There was no statistically significant difference in the height (coronal plane $=20.7 \pm 9.0 \mathrm{~mm}$ in NFAs, $20.7 \pm 11.2 \mathrm{~mm}$ in PRLomas, $p>0.05$ ), width (coronal plane $=19.4 \pm 6.4 \mathrm{~mm}$ in NFAs, $19.4 \pm 9.3 \mathrm{~mm}$ in PRLomas, $p>0.05$ ), or depth (sagittal plane $=17.3 \pm 6.3 \mathrm{~mm}$ in NFAs, $17.3 \pm 7.5 \mathrm{~mm}$ in PRLomas, $p>0.05)$ in the two tumor groups. There was also no significant difference between the mean craniocaudal diameter of NFAs $(22.3 \pm 9.3 \mathrm{~mm})$ and that of PRLomas $(23.2 \pm 11.3 \mathrm{~mm})(p=0.8)$ (Figure 3a). The mean tumor volumes were also similar in the two groups: $4.8 \pm 5 \mathrm{ml}$ for NFAs and $5.9 \pm 8 \mathrm{ml}$ for PRLomas 
(a)

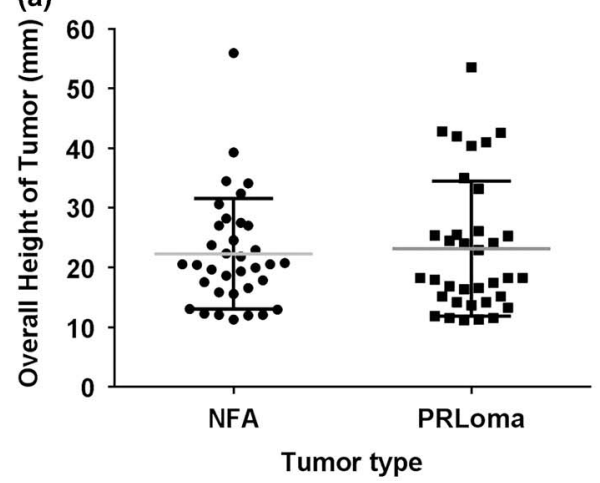

(b)

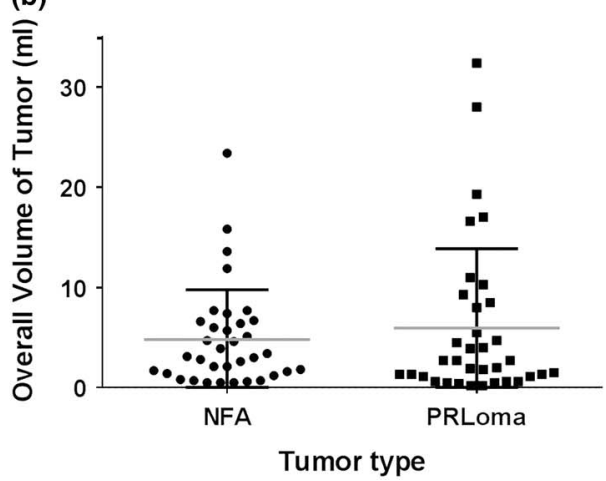

Figure 3: Height and volume comparison between NFAs and PRLomas. (a) Sagittal craniocaudal heights were similar for both NFAs and PRLomas. (b) Tumor volumes were similar for both NFAs and PRLomas.

$(p=0.47)$ (Figure 3b). The mean suprasellar growth of PRLomas $(2.9 \pm 5.3 \mathrm{~mm})$ was less than half that seen in NFAs $(7.3 \pm 4.7 \mathrm{~mm}, p<0.001)$, whereas the mean infrasellar growth of PRLomas $(10.2 \pm 8.0 \mathrm{~mm})$ was twice that observed in NFAs $(5.0 \pm 6.6 \mathrm{~mm}, p=0.04)$. A comparison of the suprasellar and infrasellar growth patterns of NFAs and PRLomas is depicted in Figure 4, which shows preferential infrasellar growth of PRLomas compared with NFAs. In our group of 70 patients, NFAs with any suprasellar growth can be detected with a sensitivity, specificity, PPV, and NPV of 97.1, 68.6, 75.6, and 96\%, respectively; whereas PRLomas with any infrasellar growth can be identified with a sensitivity, specificity, PPV, and NPV of 100, $31.4,59.3$, and $100 \%$, respectively. Similarly, NFAs with preferential suprasellar growth (difference between supra- and infrasellar growth of $>0 \mathrm{~mm}$ ) can be detected with a sensitivity, specificity, PPV, and NPV of 82.9, 94.3, 93.5, and 84.6\%, respectively; whereas PRLomas with preferential infrasellar growth (difference between infra- and suprasellar growth of $>0 \mathrm{~mm}$ ) can be identified with a sensitivity, specificity, PPV, and NPV of $94.3,82.9,84.6$, and $93.5 \%$, respectively.

\section{Visual Field Abnormalities}

All patients underwent VF testing at diagnosis. In the NFA group at presentation, 20/35 $(57.1 \%)$ patients had abnormal VF testing related to tumor growth, 13/35 (37.1\%) had normal VF tests, and 1/35 (2.8\%) had abnormal VF testing that was deemed unrelated to tumor growth. In the PRLoma group, 4/35 (11.4\%) had abnormal VF tests that were deemed related to the tumor, while $2 / 35$ $(5.7 \%)$ had abnormal VF tests that were thought to be unrelated to the tumor (VF defect related to diabetic retinopathy, and the tumor did not abut the optic chiasm), whereas 29/35 (82.8\%) had normal VF testing on initial presentation. The risk of VF abnormalities at presentation was five times higher in the NFA group $(57.1 \%)$ versus the PRLoma group $(11.4 \%)(p<0.001)$. The average tumor volume at presentation that was associated with VF abnormalities was significantly larger in PRLoma patients $(23.5 \pm 7.0 \mathrm{ml})$ than in NFA patients $(6.3 \pm 6.0 \mathrm{ml} ; p<0.001)$ (Figure 5).

\section{DISCUSSION}

Extrasellar growth is common, as most PAs present as macroadenomas. ${ }^{1}$ It has been previously reported that growth hormone
(GH)-producing (somatotrophic) tumors have a predilection for infrasellar growth. ${ }^{12}$ However, to date, no study has systematically assessed the patterns of growth of prolactin-producing tumors (lactotrophic tumors) in comparison with NFAs or assessed the clinical impact of these growth patterns on vision loss.

It is not clear from the single previous study reporting a predilection for infrasellar growth of $\mathrm{GH}$ whether the tumors were purely somatotrophic or mixed somatolactotrophic tumors. Since $\mathrm{GH}$ adenomas can also stain for and co-secrete prolactin, it is crucial to examine distinct growth patterns of PA based on specific tumor types. Consequently, in this study, we excluded those PRLomas that were associated with clinical or biochemical evidence of excessive GH production.

Defining infrasellar growth on imaging, particularly in large tumors where the sellar floor may be completely eroded, can be subjective. To make the assessment of tumor more objective, we developed a method to determine the predominant growth pattern, which can be easily done in day-to-day clinical practice on a single sagittal image. We used the planum sphenoidale as our bony landmark. Even in the largest of the tumors in our series, the planum sphenoidale remained intact.

Our study showed that, despite having similar tumor volumes and craniocaudal measurements to NFAs, PRLomas predominantly grow inferiorly. This is an intriguing finding with a potentially important clinical implication. Due to predominant inferior growth, PRLomas are highly unlikely to cause VF abnormalities unless they are very large tumors. In our study, PRLoma patients who presented with VF abnormalities had a significantly larger tumor volume $(23.5 \pm 7.0 \mathrm{ml})$ than patients with NFAs $(6.3 \pm 6.0 \mathrm{ml})(p<0.001)$. This finding may be of particular relevance in untreated pregnant women with large PRLomas who, based on recent guidelines, are generally followed during pregnancy through VF testing. ${ }^{13}$ Our data suggest that VF testing may not predict tumor growth in the absence of significant superior extension or massive enlargement of the tumor. Whether PRLomas during pregnancy also follow a similar growth pattern remains to be assessed.

The cause of these superiorly and inferiorly oriented growth patterns is not obvious. Our knowledge of PA precursors suggests that the growth patterns are unlikely to result from a more superior location of the NFA precursors and a contrastingly inferior location of PRLoma tumor precursors. NFAs are a mixture of silent 


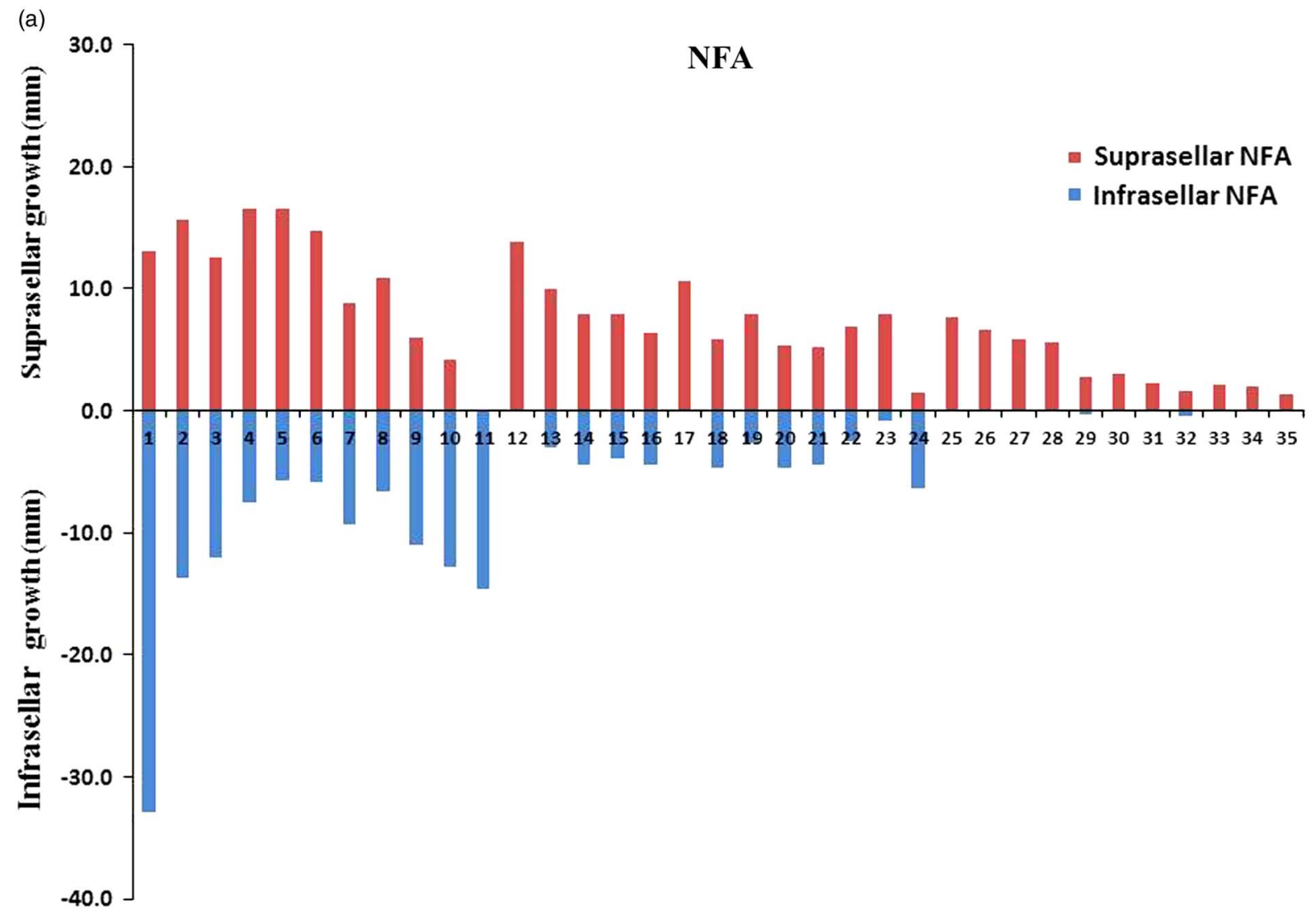

(b)

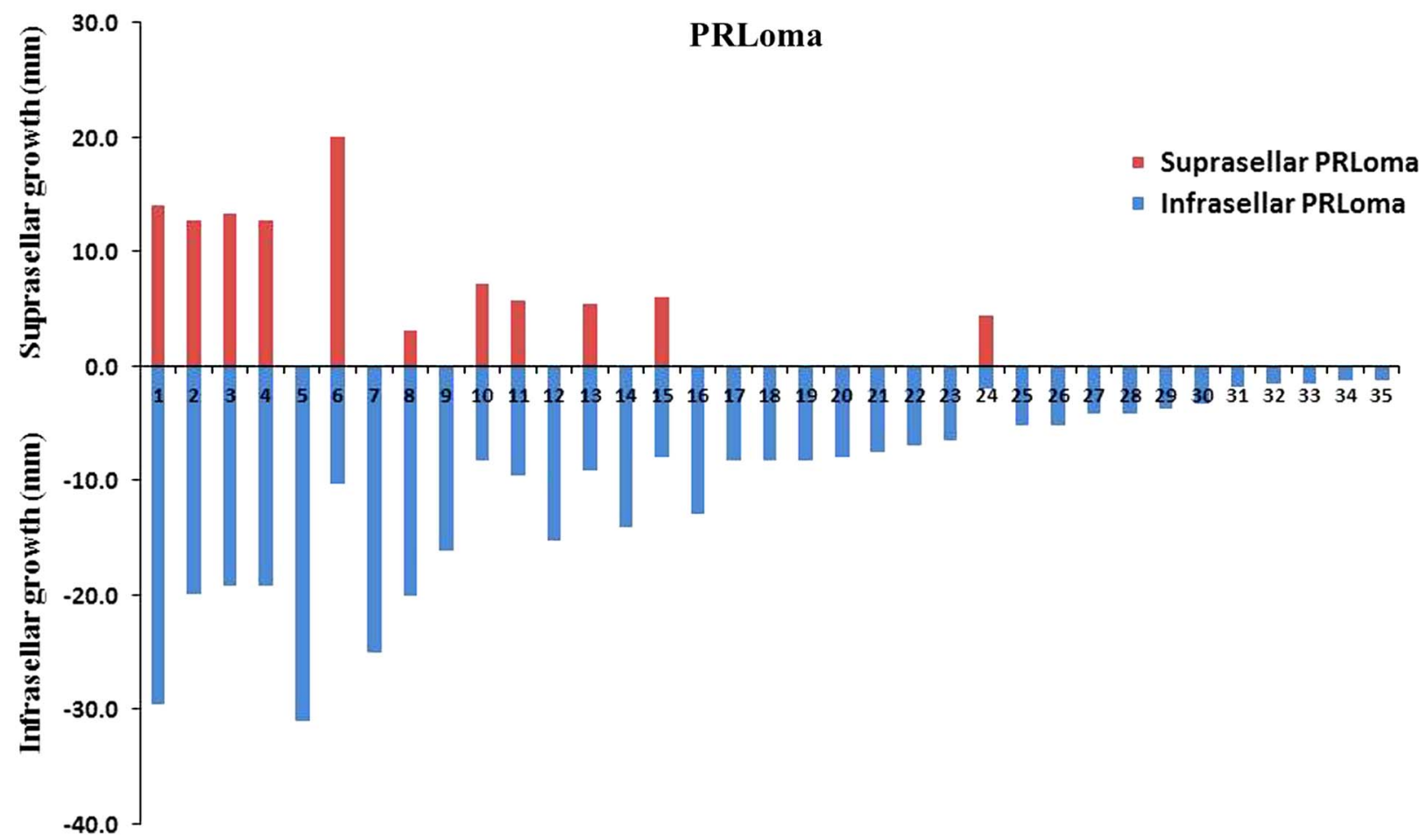

Figure 4: Graphs showing the preferential suprasellar growth in NFAs (a) compared with predominantly infrasellar growth in PRLomas (b). Tumors are ordered on the $\mathrm{X}$-axis from largest to smallest sagittal craniocaudal heights $(\mathrm{n}=35$ patients). 


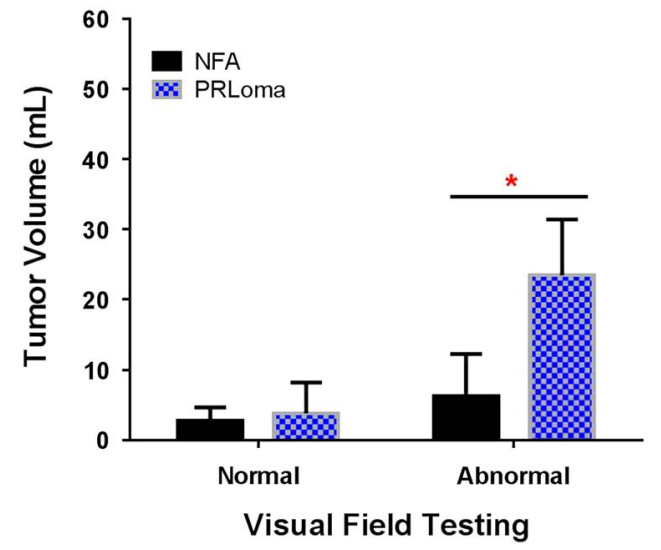

Figure 5: Visual field deficits were common in the NFA group $(\mathrm{n}=20)$; in the PRLoma group, visual field defects were less common $(\mathrm{n}=4)$ and, as shown here, occurred when the tumors were much larger. p $<0.001$.

corticotroph, somatotroph, lactotroph, thyrotroph, and gonadotroph, and are true null cell tumors. ${ }^{14}$ As such, they are thought to arise from precursors scattered throughout the anterior pituitary. Similarly, lactotrophs that are precursors of PRLomas constitute $10-30 \%$ of pituicytes and are also widely distributed throughout the pituitary. ${ }^{15}$

Given the observations of our study, it is also possible to hypothesize that there are trophic influences causing these tumors to grow preferentially in different directions. The preferential infrasellar growth of PRLomas demonstrated in this study suggests a greater ability of these tumors to erode through the bone of the sellar floor/clivus. To date, there is little in-vivo or in-vitro biochemical data concerning factors that might induce directional growth of pituicytes. Whatever the pathophysiology underlying these growth patterns, they remain of clinical importance for the treatment of patients with PAs. As such, further investigation to understand these mechanisms is warranted. This is the first study to demonstrate the inferior growth pattern of PRLomas as compared with NFAs and to assess the effects of growth pattern on vision.

\section{Conclusions}

The different growth patterns of NFAs and PRLomas are of significant clinical relevance in determining the risk of vision loss associated with different types of PA. The results of our study show that, in large NFAs and PRLomas of similar sizes, suprasellar growth and vision loss are more common in patients with NFAs. In contrast, PRLomas have preferential infrasellar growth and affect vision when their suprasellar growth is much greater than that in NFAs. These results also question the sensitivity of VF assessment in determining PRLoma growth in medically untreated patients. Additional studies are required to identify the underlying reason for this difference in growth characteristics.

\section{Abbreviations}

$\mathrm{MRI}=$ magnetic resonance imaging; $\mathrm{NFA}=$ nonfunctioning adenoma; $\mathrm{PA}=$ pituitary adenoma; $\mathrm{PRLoma}=$ prolactinoma; $\mathrm{TSS}=$ transsphenoidal surgery; $\mathrm{VF}=$ visual field.

\section{Disclosures}

Syed Ali Imran, Jai Shankar, Andrea Hebb, Sidney Croul, and David Clarke hereby state that they have nothing to disclose.

\section{Author Contributions}

Drs. Imran, Shankar, and Clarke were involved in conceptualization, data collection, data analysis, manuscript writing, and review. Dr. Hebb was responsible for data analysis and presentation as well as manuscript preparation and review. Dr. Croul was involved in pathological analysis of tumor tissue, manuscript preparation, and review. All authors approved the final version of the manuscript.

\section{REFERENCES}

1. Al-Dahmani K, Mohammad S, Imran F, et al. Sellar masses: an epidemiological study. Can J Neurol Sci. 2016;43(2):291-7.

2. Raappana A, Koivukangas J, Ebeling T, Pirilä T. Incidence of pituitary adenomas in Northern Finland in 1992-2007. J Clin Endocrinol Metab. 2010;95(9):4268-75.

3. Daly AF, Rixhon M, Adam C, Dempegioti A, Tichomirowa MA, Beckers A. High prevalence of pituitary adenomas: a crosssectional study in the province of Liege, Belgium. J Clin Endocrinol Metab. 2006;91(12):4769-75.

4. Dhasmana R, Nagpal RC, Sharma R, Bansal KK, Bahadur H. Visual fields at presentation and after trans-sphenoidal resection of pituitary adenomas. J Ophthalmic Vis Res. 2011;6 (3):187-91.

5. Grochowicki M, Khalfallah Y, Vighetto A, Berquet S, Sassolas G. Ophthalmic results in patients with macroprolactinomas treated with a new prolactin inhibitor CV 205-502. Br J Ophthalmol. 1993;77(12):785-8.

6. Alkherayf F, Edem I, Ouattara JM, Lamothe A, Agbi C. Planumclival angle classification: a novel preoperative evaluation for sellar/parasellar surgery. J Neurol Surg B Skull Base. 2015; 76(4):316-22.

7. Silverman FN. Roentgen standards for size of the pituitary fossa from infancy through adolescence. Am J Roentgenol Radium Ther Nucl Med. 1957;78(3):451-60.

8. Chilton LA, Dorst JP, Garn SM. The volume of the sella turcica in children: new standards. AJR Am J Roentgenol. 1983; 140(4):797-801.

9. Choi WJ, Hwang EH, Lee SE. The study of shape and size of normal sella turcica in cephalometric radiographs. Korean J Oral Maxillofac Radiol. 2001;31(1):43-9.

10. Taveras JM, Wood EH. Diagnostic Neuroradiology. Baltimore: Williams \& Wilkins; 1964: P. 1096-104.

11. Karpagam S, Gowri S. Brain tumor growth and volume detection by ellipsoid-diameter technique using MRI data. IJCSI Int J of Comput Sci. 2012;9(2):121-26.

12. Zada G, Lin N, Laws ER Jr. Patterns of extrasellar extension in growth hormone-secreting and nonfunctional pituitary macroadenomas. Neurosurg Focus. 2010;29(4):E4.

13. Melmed S, Casanueva FF, Hoffman AR, et al. Diagnosis and treatment of hyperprolactinemia: an Endocrine Society clinical practice guideline. J Clin Endocrinol Metab. 2011; 96(2):273-88.

14. Asa SL. Tumors of the pituitary gland. In: Atlas of Tumor Pathology. Washington, DC: Armed Forces Institute of Pathology; 2011: P. 120-35.

15. Asa SL, Penz G, Kovacs K, Ezrin C. Prolactin cells in the human pituitary: a quantitative immunocytochemical analysis. Arch Pathol Lab Med. 1982;106(7):360-3. 